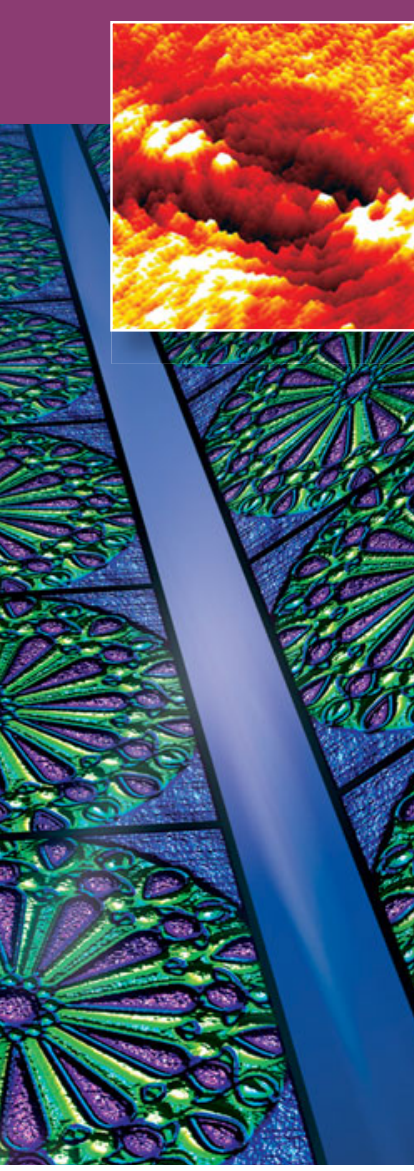

\title{
Plasmonics: Metal-worthy methods and materials in nanophotonics
}

\author{
Jennifer A. Dionne and Harry A. Atwater, Guest Editors
}

\begin{abstract}
Electrons and photons can coexist as a single entity called a surface plasmon-an elementary excitation found at the interface between a conductor and an insulator. Because of their hybrid electric and photonic nature, plasmons allow photons to be precisely controlled on the nanoscale. Plasmons are evident in the vivid hues of rose windows, which derive their color from small metallic nanoparticles embedded in the glass. They also provide the basis for color-changing biosensors (such as home pregnancy tests), photothermal cancer treatments, improved photovoltaic cell efficiencies, and nanoscale lasers. While surface plasmons were first identified nearly 55 years ago, many of their exciting applications are yet to come. This issue of MRS Bulletin reviews the progress and promise of plasmonics - from the characterization tools that have allowed nanometer-scale probing of plasmons to the new materials that may enable low-loss, active, and quantum plasmonics. Within reach are applications ranging from integrated plasmonic circuits for nanophotonic computation to plasmonic optical tweezers for manipulation of nano-sized particles and proteins.
\end{abstract}

\section{Introduction}

Discoveries of the mid-20th century have helped shape the character and quality of our modern technological landscape. The 1940s and 1950s brought remarkable breakthroughs in materials science ranging from the theory of dopants to the first imaging of individual atoms and identification of the double-helix structure of DNA. This era also produced the first transistor, the first silicon solar cell, and development of the first lasers. New discoveries about materials properties enabled new devices that in turn provided more insight into the fundamental nature of materials.

Around this time, a graduate student at the University of Tennessee-Rufus Ritchie—-began researching the energy losses of electrons passing through metallic films. Ritchie was fascinated by then recent experiments indicating that the energy loss spectrum was characterized by several equally spaced sharp lines-implying that electrons lose their energy in integral multiples of some fundamental unit. The results were not consistent with a free-particle treatment, in which the motion of electrons in a metal is assumed to be independent of all other electrons. David Pines and David Bohm at Princeton suggested that the energy loss spectra might be due to collective oscillations of the electron plasma in the metal..$^{1,2}$ Ritchie coined this new elementary oscillation a "plasmon" and began to explore its energy and angle-dependent properties. ${ }^{3}$ By further investigating the effect of the film thickness, Ritchie accounted for depolarization effects near surfaces and provided the first analysis of "surface plasmons." His theoretical work laid the foundation for what is now the thriving field of plasmonics. ${ }^{5-8}$

As defined by Ritchie, surface plasmons are collective oscillations of conduction electrons at the interface between a conductor and non-conductor. Whether excited by electrons or photons, plasmons represent a "hybrid" electron-photon mode confined to a conductor; the electrons oscillate with an amplitude and phase reminiscent of light waves, but are bound to a conducting surface. Because of the dual electronic and photonic nature of surface plasmons, they are characterized by extremely high electric field intensities and extremely small mode wavelengths. Compared to electromagnetic waves in vacuum, plasmonic fields can be enhanced by more than two orders of magnitude. ${ }^{9}$ Further, their modal volumes can be reduced by more than two orders of magnitude. ${ }^{10}$ These two properties - extreme field enhancement and confinementrender plasmons a fascinating playground for photonics, allowing optical signals to be controlled on length scales comparable to electronic wavelengths.

Since their discovery in 1957 , surface plasmons have been exploited in applications as diverse as single-molecule surface-enhanced Raman spectroscopy, ${ }^{11-13}$ nanoscale optical modulators, ${ }^{14,15}$ high-efficiency solar cells, ${ }^{16,17}$ improved 
photocatalysts, ${ }^{18-20}$ nanoscale lasers, ${ }^{21-24}$ biological rulers, ${ }^{25,26}$ and electromagnetic metamaterials for invisibility and sub-diffractionlimited optical microscopy. ${ }^{27-31}$ The explosive growth of the field is reflected in the scientific literature: since 1990, the number of papers related to surface plasmons has nearly doubled every five years. Advances in nanofabrication and characterization have accelerated this growth. Techniques such as electron beam lithography and focused ion beam milling have allowed complex plasmonic architectures to be fabricated with nanometer-scale precision. In parallel, sophisticated chemical synthetic techniques have enabled "bottom-up" assembly of diverse plasmonic nanoparticle shapes, sizes, and morphologies. Breakthroughs in electron microscopy and spectroscopy, including aberration-corrected transmission electron microscopy and cathodoluminescence imaging as well as in photon scanning tunneling microscopy, have allowed characterization of electric and magnetic plasmonic fields with near atomic-scale resolution. The ability to create and characterize complex plasmonic structures provides a powerful yet precise handle of photons in the nanoworld.

As surface plasmons celebrate their 55th anniversary, this issue of MRS Bulletin highlights the progress and promise of plasmonics. Attention is given to breakthroughs following the May 2005 MRS Bulletin issue on "Synthesis and Plasmonic Properties of Nanostructures," 32 including

- Integrated plasmonic circuits for subwavelength optical communications and computation

- Plasmonic tweezers for light-controlled mechanical manipulation of nanoparticles and biomolecules

- Development of tools to image plasmonic fields with sub-5-nm resolution, using advanced electron microscopy, spectroscopy, and super-resolution microscopy

- The emergence of quantum plasmonics, where the quantum nature of light and/or electrons leads to properties unique from a classical description

- Loss compensation in plasmonic devices using novel materials such as transparent conducting oxides, transition metal nitrides, semiconductors, and graphene.

The discoveries, challenges, and prospects discussed in each article imply a thriving golden era and bright future for plasmonics.

\section{Passive and active plasmonic devices}

While surface plasmons were first identified on metallic films, there are a number of geometries that support surface plasmons. Figure $1(\mathrm{a}-\mathrm{c})$ illustrates three plasmonic structures that form the basis for many plasmonic devices, including a planar film, a coaxial pillar, and a nanoparticle. These structures highlight the key distinctions between propagating, localized, and hybrid plasmons and also provide a framework for understanding more complex plasmonic architectures.

A planar conducting film supports surface plasmons that propagate as transverse-magnetic waves along its interface with an insulator. ${ }^{15,116}$ As seen in Figure 1a, electric fields are maximized at the conductor-insulator interface, but they decay evanescently in the perpendicular direction - usually within a few tens to hundreds of nanometers. Since the momentum of plasmons on planar films usually exceeds that of photons in free-space, a prism, grating, groove, or scatterer must be used to provide photons with sufficient momentum to excite surface plasmons. Figure 1d illustrates plasmon propagation along a single gold interface, excited using $514 \mathrm{~nm}$ light incident on an elliptical groove. ${ }^{33}$ Here, a thin, electric-field-sensitive photoresist on the gold was used to record plasmonic fields as topographic changes that could later be imaged with atomic force microscopy. Outside of the groove, the plasmon decays within a few microns in the direction of propagation. Within the ellipse, the surface plasmon forms a standing wave that maps the allowed modes and foci of the ellipse. The ripples in the resist correspond to a surface plasmon wave with a wavelength of $438 \mathrm{~nm}$ - about $15 \%$ smaller than the wavelength of the incident light. Depending on the film material, thickness, and surrounding media, plasmon wavelengths can be tuned down to tens of nanometers, while propagation lengths can exceed millimeters. Their sensitivity to these parameters - particularly the index of the surrounding media-has rendered planar 
plasmonic architectures as efficient biological sensors ${ }^{34}$ and optical modulators. ${ }^{35}$

In addition to propagation along a single conducting interface, plasmons can also propagate within narrow dielectric channels. For example, a shallow groove in a metallic film supports "channel" plasmon polariton modes that propagate along the dielectric region with minimal decay into the surrounding metal. ${ }^{36,37}$ Similarly, metal-insulator-metal or semiconducting-insulator-metal multilayers can support "gap" $38-40$ or "hybrid" 41 plasmon polariton modes. Akin to linear combinations of atomic orbitals, these modes resemble linear combinations of single-interface plasmonic modes. For example, Figure 1b illustrates a coaxial plasmonic geometry composed of a metal-insulator-metal multilayer structure with cylindrical symmetry. ${ }^{41,42}$ As with the single metallic interface geometry, plasmons in the coax propagate along the metal-dielectric interface, with maximum field intensities at the metal-dielectric boundary. However, unlike single interface plasmons, the field intensities within the dielectric channel can be much larger, and these gap plasmons propagate with a much higher degree of confinement. Interestingly, the metallic and semiconducting layers in multilayer plasmonic structures not only serve to confine plasmons, but can also double as electrical contacts. Such structures have been implemented as efficient electrooptic modulators, ${ }^{43,44}$ as interconnects for short-range optical data transmission, ${ }^{45}$ and also as gradient-index media (including negative index media). ${ }^{46}$ Multilayer plasmonic resonators have also formed the basis for room-temperature nanoscale lasers, some capable of achieving thresholdless lasing. ${ }^{21-24}$

As seen in Figure 1c, a conducting nanoparticle with dimensions much smaller than the wavelength of light exhibits a purely localized plasmon resonance, reminiscent of an electric dipole. ${ }^{117}$ Its scattering spectrum depends sensitively on the particle size, shape, charge, and the surrounding dielectric media. For example, Figure 1e illustrates a dark-field optical micrograph of silver nanoparticles of various sizes (10-100 nm); while smaller particles preferentially scatter toward the blue spectral regime, larger particles scatter preferentially toward the red. The strong local resonances supported by plasmon nanoparticles have been utilized in biological and catalytic sensing, ${ }^{20,34}$ as well as in "spasing" - surface plasmon amplification by stimulated emission of radiation. ${ }^{47,48}$ Such spasers represent the smallest surface plasmon lasers to date. Further, since nanoparticle extinction cross-sections are usually much larger than the physical particle size, plasmon nanoparticles serve as efficient receiving and transmitting "nanoantennas" for optical signals. ${ }^{4-52,118}$ Most recently, plasmon nanoparticles have been implemented as novel photodetectors based on hot-electron injection into a semiconductor ${ }^{53}$ and as optical rectifiers that convert optical fields into dc electrical power via resonant plasmon absorption. ${ }^{54}$

The article by Sorger and colleagues in this issue discusses integration of such passive and active plasmonic components for on-chip plasmonics. Particular attention is given to plasmonic interconnects, plasmonic sources (such as lasers, spasers, and light-emitting diodes), modulators (including alloptical and electro-optical switches), and detectors based on planar, hybrid, or nanoparticle plasmon modes. Like photonic circuits, plasmonic circuits promise higher bandwidth, higher speed, lower latency, and reduced power consumption than electronic circuits. ${ }^{55}$ Plasmonic circuits also promise miniaturization of photonic circuits - with deeply subwavelength dimensions and dense component integration commensurate with electronic circuits. Such an integrated plasmonic technology could revolutionize the speed, size, cost, and power requirements of modern computational networks, provided key challenges such as loss and inter-device coupling can be addressed.

\section{A strong hold on nano-objects: Plasmonic optical tweezers}

Plasmonic components allow for precise sculpting of electromagnetic fields on deeply subwavelength scales. Beyond applications to optical communication and computation, this property is a key enabler for optical tweezing - the trapping and manipulation of objects using radiation pressure. Conventional optical tweezers rely on gradient forces within a diffractionlimited laser focus. ${ }^{56}$ Their ability to manipulate small objects with light has enabled unprecedented opportunities in the biosciences. For example, optical tweezers have allowed for non-invasive manipulation of individual living cells, ${ }^{57}$ as well as a deeper understanding of molecular motors. ${ }^{58}$ However, because optical trapping forces in the Rayleigh regime scale with the cube of the object radius,${ }^{59}$ conventional optical trapping of submicron-sized particles is challenging-requiring either an increase in the local field intensity or a strong reduction of the potential well width. Plasmonic tweezers address both requirements, enabling high local electromagnetic field intensities in subwavelength volumes. ${ }^{60,61}$ As described in the article by Quidant, plasmonic nanostructures can act as a "nanolens" capable of concentrating light beyond the capabilities of a microscope objective. Accordingly, plasmonic components allow nanoscale objects to be trapped and manipulated with much smaller optical powers than would be required with conventional optical tweezers.

Plasmonic tweezers were first realized using a planar metallic film, as in Figure 1a. The largely increased electric field intensities at the metal-dielectric interface provided a nearly 40 -fold enhancement in the trapping forces compared to conventional optical tweezers. ${ }^{62}$ More recently, plasmonic tweezers have been implemented with plasmonic nanoantennas, as shown in Figure 2. A single subwavelength Au nanorod produces plasmon hot spots (i.e., regions of strong electromagnetic fields) that result in an $\sim 10-k_{\mathrm{B}} T$-deep potential well (where $k_{\mathrm{B}}$ is Boltzmann's constant, and $T$ is the temperature). ${ }^{60}$ The well depth and full-width-at-half-maximum can be tuned by varying the antenna geometry. For example, gap nanoantennas composed of two closely spaced metallic nanoparticles have been used to trap $200 \mathrm{~nm}$ polystyrene beads, living Escherichia coli bacteria, and $10 \mathrm{~nm}$ Au nanoparticles. ${ }^{63,64}$ Plasmonic nanoantennas have 


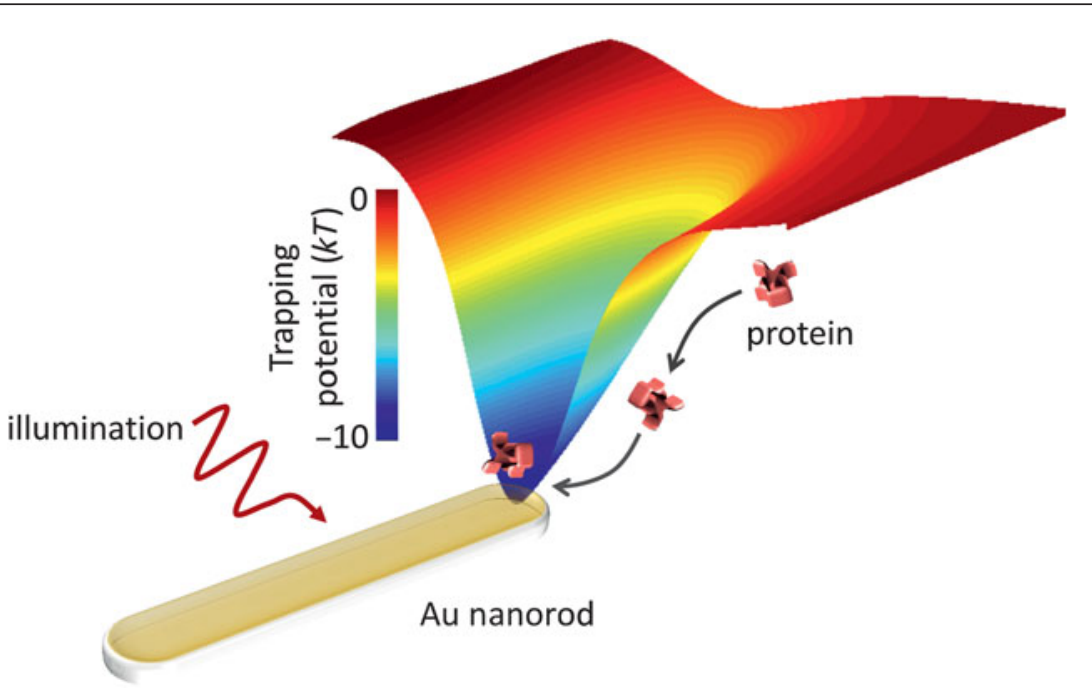

Figure 2. Plasmon nanoantennas, such as metallic nanorods, have been used as optical tweezers for trapping and manipulating nanoscale objects ranging from $\sim 10 \mathrm{~nm}$ particles to proteins. Their highly localized electromagnetic fields produce deep trapping potentials in subwavelength volumes. by a Gaussian curve with a diffraction-limited full-width at half-maximum, the centroid of the emission defines the observed signal position. Provided no other emitters are nearby, this centroid position can be calculated to better than $5 \mathrm{~nm}$ - significantly better than the diffraction limit of light. ${ }^{70-72}$ The article by Weber and Willets describes application of such superresolution imaging techniques to mapping plasmonic hot spots and tracking the motion of single molecules near plasmonic nanostructures. For example, Figure 3a illustrates the surface-enhanced Raman scattering (SERS) spectral signal from a single R6G molecule near an Ag nanoparticle dimer. ${ }^{73,74}$ The SERS spatial intensity map — plotted via a color map - is overlayed onto a scanning electron micrograph of the dimer. Using super-resolution techniques, the molecule's motion and SERS intensity can be tracked in real-time, providing insight into how molecules explore plasmonic hot spots. Complementarily, single-molecule fluorescence

also been used to rotate particles by rotation of the incident polarization..$^{65}$

Currently, research in plasmonic tweezers is aimed at trapping and manipulating much smaller particles, in the sub-10-nm regime. An important advance in this area has been the development of self-reconfigurable (or self-induced back action) traps; these plasmonic tweezer designs dynamically adjust the field intensity and the trap position to compensate for Brownian motion of the particle. To date, such plasmonic traps have enabled trapping of $20 \mathrm{~nm}$ polystyrene beads and large proteins (dimensions of $\sim 10 \mathrm{~nm}$ ). ${ }^{66,67}$ More sophisticated optical plasmonic traps are likely to lead to trapping of single, sub-10-nm particles and proteins ${ }^{68,69}$ and perhaps, ultimately, even single atoms.

\section{Nanometer-scale imaging and characterization of plasmons}

The fidelity with which plasmonic components can be used to manipulate optical signals relies in large part on our ability to characterize plasmonic fields. Two pivotal developments of the past decade have enabled characterization of plasmonic modes with sub-5-nm resolution: super-resolution microcopy and electron spectroscopy. While both tools are complementary to near-field optical techniques (which detect evanescent fields and provide a resolution of $\sim 15 \mathrm{~nm}$ ), super-resolution microscopy and electron spectroscopy can provide insights into dynamic plasmon processes, often with the ability to resolve polarization and angle-dependent profiles.

Super-resolution microscopy techniques — such as PALM (photoactivated localization microscopy) and STORM (stochastic optical reconstruction microscopy) — use the fluorescence signal from isolated emitters to achieve sub-diffraction-limited resolution. While the emission of a fluorophore is characterized can be used to accurately map the near-fields of plasmonic structures with $\sim 5-\mathrm{nm}$ resolution. ${ }^{75,76}$

Electron-based techniques have also emerged as powerful probes of plasmonic modes. The article by Vesseur et al. and electron energy loss spectroscopy (EELS) to achieve near atomic-scale resolution of plasmonic fields. In both techniques, an electron incident on a nanostructure acts as a white-light or broadband point-source that excites plasmonic modes. ${ }^{77}$ EELS measurements rely on detection of electrons transmitted through a plasmonic structure, similar to Ritchie's first surface plasmon measurements; peaks in the energy loss spectrum correspond to excitation of surface and bulk plasmonic modes. ${ }^{3,78}$ Recent advances in monochromated and aberration-corrected transmission electron microscopy have allowed for unprecedented imaging ( $<0.3 \mathrm{~nm}$ resolution) and spectroscopy of plasmonic components, recently with ultrafast time resolution (see Figure $3 b){ }^{79-82}$

In contrast, CL measurements rely on photons emitted by the excited modes upon radiative relaxation; these photons can then be detected in the far-field of a scanning electron microscope. ${ }^{83,84}$ By scanning the electron source and the detector wavelength, full maps of plasmonic modes can be obtained across the visible and near-infrared spectrum. Recent advances in CL have also enabled reconstruction of angle- and polarizationdependent plasmon emission. ${ }^{85}$ The resolution of CL imaging spectroscopy is limited only by the scanning electron microscope, with sub-5-nm accuracy readily obtained. For example, Figure $3 \mathrm{c}-\mathrm{d}$ illustrates a scanning electron micrograph of a plasmonic ring resonator (a 2.6 micron Au disc surrounded by 15 concentric grooves) and its corresponding CL image, acquired by collecting photons of wavelength $661 \mathrm{~nm} .{ }^{86} \mathrm{~A}$ plasmonic standing wave with multiple antinodes (i.e., maxima) discusses cathodoluminescence (CL) imaging spectroscopy 


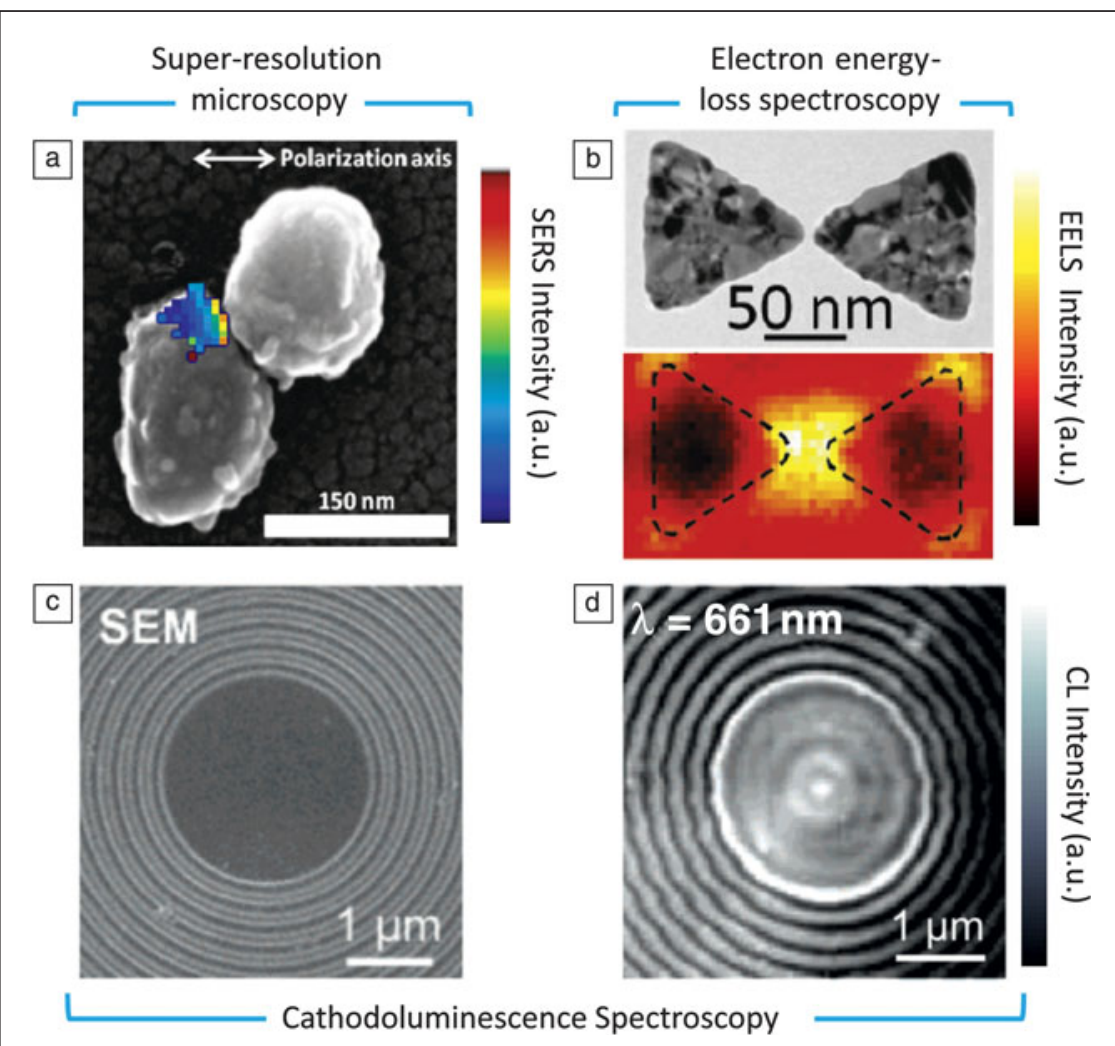

Figure 3. Advances in optical and electron microscopy allow plasmonic systems to be probed with nanometer-scale resolution. (a) Correlated super-resolution microscopy and electron microscopy of surface-enhanced Raman hot spots in a nanoparticle dimer. Reprinted with permission from Reference 73. (02011, American Chemical Society. (b) Correlated transmission electron microscopy and electron energy-loss spectroscopy (EELS) of a plasmonic mode in a silver nanoantenna. Adapted with permission from Reference 80. (02011, American Chemical Society. The EELS map was acquired for electron energies of $\sim 1.5 \mathrm{eV}(\lambda=828 \mathrm{~nm})$. (c-d) Correlated scanning electron microscopy (SEM) and cathodoluminescence (CL) spectroscopy of a silver plasmonic nanoresonator. Adapted with permission from Reference 86. (02007, American Chemical Society. The CL map was acquired for photon energies of $\sim 1.9 \mathrm{eV}(\lambda=661 \mathrm{~nm})$. SERS, surface-enhanced Raman scattering.

forces can be engineered, opening the door to complementary characterization and assembly of complex plasmonic nanostructures.

\section{Toward quantum plasmonics}

In recent years, the finesse with which plasmonic systems can be explored has entered the quantum realm. Here, properties such as entanglement, wave-particle duality, tunneling, and discretized energy levels emerge. For example, it was recently shown that two photons remain entangled upon conversion to plasmons, even though plasmons involve a macroscopic number of conduction electrons. ${ }^{94-96}$ In other words, plasmons behave as a single quantum entity. Related measurements demonstrated that plasmons can coherently exist at different times separated by much longer periods than the plasmon lifetime, due to the energy-time uncertainty principle. ${ }^{96}$ Double-slit measurements and plasmon self-interference indicate that plasmons behave as waves. ${ }^{97,98}$ But plasmons can also behave as particles, as evidenced by antibunching experiments of coupled $\mathrm{Ag}$ nanowire/diamond NV-center emitters. ${ }^{98}$

The article by Jacob explores the exotic properties of quantum plasmons, evident when either the particle nature of photons or the wave nature of electrons emerges. Attention is first given to quantum light - the interaction of plasmonic systems with nonclassical light sources (i.e., single photon emitters.) Based on the broadband tunability of the LDOS and Purcell factor, plasmons can significantly increase the efficiency of single photon sources. Such an effect has

is clearly observed within the resonator. The image implies that plasmon resonances in CL are most efficiently excited at the antinodes of the electric field - indicating that the electron beam couples to plasmonic modes via the local density of optical states (LDOS) ${ }^{87}$ Therefore, CL imaging spectroscopy can be used to map the photonic and plasmonic LDOS with nanometer-scale resolution. CL techniques can also be used to map plasmon dispersion, propagation, and emission profiles. To date, the technique has been applied to systems ranging from single metallic nanoparticles and nanowires to plasmonic whispering gallery cavities, metal-insulator-metal waveguide (MIM) nanoantennas, and plasmonic metamolecules. ${ }^{88-91}$

Electron beams can also induce forces, similar to plasmonic tweezers. The article by Vesseur et al. discusses new techniques to manipulate particles within an electron microscope, exploiting the interaction between plasmonic fields and the incoming electron. This "nanobilliards" approach has recently been used to move single, nanometer-scale Au nanoparticles in a transmission electron microscope. ${ }^{92,93}$ Both attractive and repulsive been observed in metallic structures and is predicted to be even stronger in novel plasmonic systems based on graphene. ${ }^{99-101}$ The article also discusses quantum matter-where the wave nature of matter manifests itself in localized plasmon resonances. For example, surface plasmons in nanoparticles less than approximately $5 \mathrm{~nm}$ in diameter exhibit quantum behavior reflecting the discretized, quantum nature of conduction electron transitions. ${ }^{82}$ Similarly, closely spaced metal nanoparticles are predicted to exhibit quantum tunneling that shifts the plasmon resonance from a classical description, as illustrated in Figure 4a. ${ }^{102}$ Quantum effects can also emerge in metamaterials - artificially engineered materials with vastly tunable electric permittivities and magnetic permeabilities. Metamaterials with strong dielectric anisotropy exhibit singularities in the density of states and topological transitions (i.e., abrupt changes in the isofrequency surface of allowed wave vectors). ${ }^{103,104}$ Such properties increase the electromagnetic states available to emitters while simultaneously channeling single-photon emission into subwavelength dimensions. 

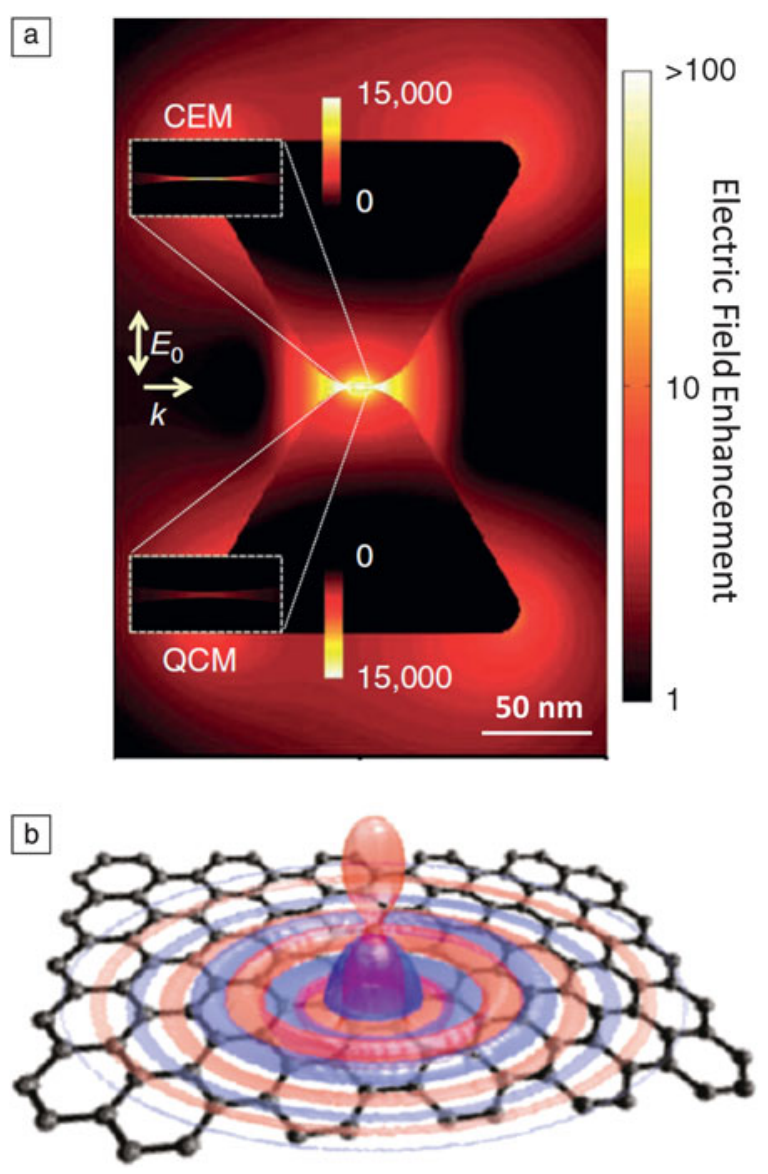

Figure 4. New plasmonic materials and properties may enable an entirely new class of plasmonic devices. (a) In the quantum realm, plasmons exhibit properties such as tunneling, entanglement, and wave-particle duality. For example, plasmon modes in a bow-tie nanoantenna will tunnel across the antenna gap. This quantum phenomenon changes the local field profile-as calculated with a quantum corrected model (QCM) - compared to a classical electromagnetic model (CEM). Reprinted with permission from Reference 102. (2011, Nature Publishing Group. (b) Doped semiconducting films, nanoparticles, and graphene may outperform noble metals as plasmonic materials and metamaterials. These materials support relatively low-loss, electrically tunable surface plasmons in the visible and infrared and can enable strong light-matter interactions. Reprinted with permission from Reference 101. (02011, American Chemical Society. In this figure, a dipole source is used to excite very short wavelength surface plasmons on a graphene film. $k$, wave vector.

While many features of quantum plasmonic systems remain unknown, numerous applications to subnanometer-scale photonics can be envisaged. For example, quantum plasmon transitions and tunneling are expected to have a significant impact on single molecule spectroscopy and biochemical sensing. Quantum plasmonic systems could increase spontaneous emission rates beyond stimulated emission, potentially enabling autofluorescence of normally weak emitters and ultrafast modulation of nano-sized light-emitting diodes. ${ }^{105}$ Further, by increasing the interaction of single photons with matter, quantum plasmonic systems could enable efficient single-photon sources, detectors, and transistors - potentially revolutionizing future quantum optical networks.

\section{Novel plasmonic materials: Overcoming loss}

Perhaps the biggest challenge facing plasmonics is loss. While metals provide the requisite negative electric permittivity for subwavelength confinement, they are also characterized by a significant imaginary component of the permittivity. This large absorption hinders long-range plasmon propagation in waveguides, challenges the development of gain-based components such as surface plasmon lasers and transistors, and serves as a significant impediment to metamaterial applications, such as electromagnetic cloaks and superlenses. In recent years, several clever experiments have addressed plasmon loss. For example, plasmon amplification and lasing have been observed in planar, cylindrical, and stripe waveguides, as well as in spasing nanoparticles. ${ }^{15}$ Moreover, gain has been introduced into resonator-based metamaterials to achieve low-loss and even active negative index media. ${ }^{106}$ While the results are encouraging, considerable pump power or operation well below room temperature is usually required. The field of plasmonics would benefit tremendously from new materials systems that could allow simultaneous subwavelength optical confinement and loss mitigation. ${ }^{107,108}$

The article by Khurgin and Boltasseva tackles this grand challenge of plasmonics, first identifying the fundamental cause of plasmonic loss and then proposing mechanisms to overcome it. By definition, surface plasmons represent coupling of an electromagnetic field to the kinetic motion of free carriers. Plasmon absorption (i.e., loss of kinetic energy) occurs through two mechanisms: (1) transitions from occupied to unoccupied states in the main $s-p$ conduction band (intraband transitions) or (2) transitions from occupied states in the $d$-band to unoccupied states in the $s-p$ band (interband transitions). The primary way to reduce absorption is to reduce the density of states available for scattering. This consideration motivates the use of highly doped semiconductors as alternatives to noble metals.

Recently, transparent conducting oxides and transition metal nitrides have received considerable attention as low-loss plasmonic materials. For example, indium tin oxide and galliumdoped zinc oxide exhibit metallic properties for wavelengths longer than 1.3 microns, while aluminum-doped zinc oxide (AZO) becomes metallic past 1.8 microns. At technologically relevant near-infrared frequencies, the losses of AZO are about four times smaller than those of Ag. ${ }^{109}$ Materials such as titanium nitride (TiN) and zirconium nitride offer visiblefrequency conductivity, becoming metallic for wavelengths longer than $500 \mathrm{~nm}$. Compared to silver and gold, TiN offers comparable performance for plasmonic applications and significantly improved performance for metamaterial applications. ${ }^{110}$ Semiconducting quantum dots have also been explored as alternatives to noble metal nanoparticles. Localized surface plasmons have been observed in semiconducting quantum dots of copper sulphide, tungsten oxide, and indium tin oxide. ${ }^{111-113}$ Further into the infrared, graphene has been shown to support 
extremely short-wavelength plasmons, with wavelengths of $\sim 200 \mathrm{~nm}$ achievable at free-space wavelengths of 10 microns (see Figure 4b). ${ }^{103,114,119}$ Unlike noble metals, the permittivity of these doped semiconductors and graphene can be vastly tuned by electrical or chemical modulation. The low loss and optical tunability of these materials could be a transformative technology, with applications ranging from integrated plasmonics to metamaterials and plasmon photovoltaics.

\section{Outlook}

Plasmonics has achieved astounding progress over the past 55 years. Born of a simple question about energy loss peaks in metallic films, plasmonics has now entered diverse application areas ranging from optoelectronics to photovoltaics, catalysis, chemistry, biology, medicine, quantum optics, and even art. A search on Google Scholar returns over 300,000 scholarly articles related to "plasmons," 18,000 of which were published since 2011. The field now has its own journal, its own Gordon conference, and has fueled several start-up companies. The articles selected for this issue represent just a small but exciting cross-section of plasmonics research. They highlight important foundational work and a vision for the future: Integrated plasmonic circuits may one day transmit petabytes of data; non-invasive gene repair might be enabled by plasmonic tweezers; plasmonic forces might enable atom-by-atom assembly; advanced plasmonic microscopies may permit subnanometerscale structural probes; and quantum plasmonic phenomena may lead to advanced quantum communication, computation, and cryptography. Undoubtedly, new discoveries about plasmonic properties will enable new devices and, in turn, provide more insight into the fundamental nature of plasmonic modes. But to paraphrase Niels Bohr, every sentence uttered must be understood not as an affirmation, but as a question. Just as plasmonics began with one spark of curiosity, we hope the articles in this issue of MRS Bulletin will inspire many more questions to come.

\section{Acknowledgments}

We are immensely grateful to all of the authors who contributed an article to this issue of MRS Bulletin. Special thanks to Amr Saleh and Sassan Sheikholeslami for providing assistance with figures; and to Jonathan Scholl, Ashwin Atre, Aitzol GarciaEtxarri, Andrea Baldi, Hadiseh Alaeian, Brian Baum, Justin Briggs, Vivian Ferry, Dennis Callahan, Matt Sheldon, Diane $\mathrm{Wu}$, and Mark Brongersma for insightful discussions. J.A.D. and H.A.A acknowledge the AFOSR, NSF, DOE, ARO, and GCEP for their generous support of research on plasmonic materials and metamaterials.

\section{References}

1. D. Bohm, D. Pines, Phys. Rev. 82, 625 (1951).

2. D. Pines, Rev. Mod. Phys. 28, 184 (1956)

3. R.H. Ritchie, Phys. Rev. 106, 874 (1957).

4. A.L. Frank, E.T. Arakawa, R.D. Birkhoff, Phys. Rev. 126, 1935 (1962).

5. W.L. Barnes, A. Dereux, T.W. Ebbesen, Nature 424, 824 (2003).

6. D.K. Gramotnev, S.I. Bozhevolnyi, Nat. Photonics 4, 83 (2010).

7. J.A. Schuller, E.S. Barnard, W. Cai, Y.C. Jun, J.S. White, M.L. Brongersma, Nat. Mater. 9, 193 (2010)
8. M.I. Stockman, Phys. Today 64, 39 (2011).

9. K. Tanabe, J. Phys. Chem. C 112, 15721 (2008).

10. H. Miyazaki, Y. Kurokawa, Phys. Rev. Lett. 96, 1 (2006).

11. M. Fleischmann, Chem. Phys. Lett. 26, 163 (1974).

12. D. Jeanmaire, R. Van Duyne, J. Electroanal. Chem 84, 1 (1977).

13. M. Moskovits, Rev. Mod. Phys. 57, 783 (1985).

14. M.I. Stockman, N.I. Zheludev, K.F. Macdonald, Z.L. Sa, Nat. Photonics 3 , 55 (2009).

15. P. Berini, I. De Leon, Nat. Photonics 6, 16 (2011).

16. H.A. Atwater, A. Polman, Nat. Mater. 9, 205 (2010).

17. A. Polman, H.A. Atwater, Nat. Mater. 11, 174 (2012).

18. S. Linic, P. Christopher, D.B. Ingram, Nat. Mater. 10, 911 (2011).

19. I. Thomann, B.A. Pinaud, Z. Chen, B.M. Clemens, T.F. Jaramillo, M.L. Brongersma, Nano Lett. 11, 3440 (2011).

20. C. Novo, A.M. Funston, P. Mulvaney, Nat. Nanotechnol. 3, 598 (2008).

21. M.T. Hill, Y.-S. Oei, B. Smalbrugge, Y. Zhu, T. De Vries, P.J. Van Veldhoven, F.W.M. Van Otten, T.J. Eijkemans, J.P. Turkiewicz, H. De Waardt, E.J. Geluk, S.-H. Kwon, Y.-H. Lee, R. Notzel, M.K. Smit, Nat. Photonics 1, 589 (2007).

22. R.M. Ma, R.F. Oulton, V.J. Sorger, G. Bartal, X. Zhang, Nat. Mater. 10, 2 (2010).

23. M. Khajavikhan, A. Simic, M. Katz, J.H. Lee, B. Slutsky, A. Mizrahi, V. Lomakin, Y. Fainman, Nature 482, 204 (2012).

24. R.F. Oulton, V.J. Sorger, T. Zentgraf, R.-M. Ma, C. Gladden, L. Dai, G. Bartal, X. Zhang, Nature 461, 629 (2009)

25. N. Liu, M. Hentschel, T. Weiss, A.P. Alivisatos, H. Giessen, Science 332 1407 (2011)

26. C. Sönnichsen, B.M. Reinhard, J. Liphardt, A.P. Alivisatos, Nat. Biotechnol. 23, 741 (2005)

27. V.M. Shalaev, Nat. Photonics 1, 41 (2007).

28. X. Ni, N.K. Emani, A.V. Kildishev, A. Boltasseva, V.M. Shalaev, Science 335 427 (2012).

29. J.A. Fan, C. Wu, K. Bao, J. Bao, R. Bardhan, N.J. Halas, V.N. Manoharan, P. Nordlander, G. Shvets, F. Capasso, Science 328, 1135 (2010).

30. Y.A. Urzhumov, G. Shvets, J. Fan, F. Capasso, D. Brandl, P. Nordlander, Opt. Express 15, 14129 (2007).

31. H. Shin, S. Fan, Phys. Rev. Lett. 96, 1 (2006).

32. Y. Xia, N.J. Halas, MRS Bull. 30, 5 (2005).

33. J.A. Dionne, PhD thesis, California Institute of Technology (2009).

34. J.N. Anker, W.P. Hall, C. Nilam, J. Zhao, P. Van, Nat. Mater. 7, 8 (2008).

35. D. Pacifici, H.J. Lezec, H.A. Atwater, Nat. Photonics 1, 402 (2007).

36. S.I. Bozhevolnyi, V.S. Volkov, E. Devaux, J.-Y. Laluet, T.W. Ebbesen, Nature 440, 508 (2006).

37. D.K. Gramotnev, D.F.P. Pile, Appl. Phys. Lett. 85, 6323 (2004).

38. J. Dionne, L. Sweatlock, H. Atwater, A. Polman, Phys. Rev. B 73, 1 (2006).

39. R. Zia, M.D. Selker, P.B. Catrysse, M.L. Brongersma, J. Opt. Soc. Am. A 21, 2442 (2004).

40. R.F. Oulton, V.J. Sorger, D.A. Genov, D.F.P. Pile, X. Zhang, Nat. Photonics 2, 496 (2008).

41. D. Waele, S.P. Burgos, A. Polman, H.A. Atwater, Nano Lett. 9 (8), 2832 (2009).

42. A.A.E. Saleh, J.A. Dionne, Phys. Rev. B 85, 1 (2012).

43. J.A. Dionne, K. Diest, L.A. Sweatlock, H.A. Atwater, Nano Lett. 9, 897 (2009). 44. W. Cai, J.S. White, M.L. Brongersma, Nano Lett. 9 (12), 4403 (2009).

45. J.A. Dionne, H.J. Lezec, H.A. Atwater, Nano Lett. 6, 1928 (2006).

46. S.P. Burgos, R. de Waele, A. Polman, H.A. Atwater, Nat. Mater. 9, 407 (2010). 47. M.A. Noginov, G. Zhu, A.M. Belgrave, R. Bakker, V.M. Shalaev, E.E. Narimanov, S. Stout, E. Herz, T. Suteewong, U. Wiesner, Nature 460, 1110 (2009).

48. M.I. Stockman, J. Opt. 12, 024004 (2010).

49. P. Alonso-González, P. Albella, M. Schnell, J. Chen, F. Huth, A. Garcia-Etxarri, F. Casanova, F. Golmar, L. Arzubiaga, L.E. Hueso, J. Aizpurua, R. Hillenbrand, Nat. Commun. 3, 684 (2012).

50. A. Kinkhabwala, Z. Yu, S. Fan, Y. Avlasevich, K. Müllen, W.E. Moerner, Nat. Photonics 3, 654 (2009).

51. A.G. Curto, G. Volpe, T.H. Taminiau, M.P. Kreuzer, R. Quidant, N.F. van Hulst, Science 329, 930 (2010).

52. A. Alù, N. Engheta, Nat. Photonics 2, 307 (2008).

53. M.W. Knight, H. Sobhani, P. Nordlander, N.J. Halas, Science 332, 702 (2011).

54. M. Sheldon, H.A. Atwater, Condens. Matter, in press (available at http://arxiv. org/abs/1202.0301).

55. J.A. Dionne, L.A. Sweatlock, M.T. Sheldon, A.P. Alivisatos, H.A. Atwater, IEEE J. Sel. Top. Quantum Electron. 16, 295 (2010).

56. A. Ashkin, J.M. Dziedzic, J.E. Bjorkholm, S. Chu, Opt. Lett. 11, 288 (1986).

57. A. Ashkin, J.M. Dziedzic, T. Yamane, Nature 330, 769 (1987).

58. C. Bustamante, Z. Bryant, S.B. Smith, Nature 421, 423 (2003)

59. P.W. Smith, A. Ashkin, W.J. Tomlinson, Opt. Lett. 6, 284 (1981).

60. M.L. Juan, M. Righini, R. Quidant, Nat. Photonics 5, 349 (2011).

61. A.N. Grigorenko, N.W. Roberts, M.R. Dickinson, Y. Zhang, Nat. Photonics 2, 365 (2008). 
62. G. Volpe, R. Quidant, G. Badenes, D. Petrov, Phys. Rev. Lett. 96, 1 (2006). 63. M. Righini, P. Ghenuche, S. Cherukulappurath, V. Myroshnychenko, F.J. García de Abajo, R. Quidant, Nano Lett. 9, 3387 (2009).

64. W. Zhang, L. Huang, C. Santschi, O.J.F. Martin, Nano Lett. 10, 1006 (2010). 65. K. Wang, E. Schonbrun, P. Steinvurzel, K.B. Crozier, Nat. Commun. 2, 469 (2011).

66. C. Chen, M.L. Juan, Y. Li, G. Maes, G. Borghs, P. Van Dorpe, R. Quidant, Nano Lett. 12, 125 (2012).

67. Y. Pang, R. Gordon, Nano Lett. 12, 402 (2012)

68. R. Sainidou, F. García de Abajo, Phys. Rev. Lett. 101, 1 (2008).

69. X. Yang, Y. Liu, R.F. Oulton, X. Yin, X. Zhang, Nano Lett. 11, 321 (2011).

70. M.P. Gordon, T. Ha, P.R. Selvin, Proc. Natl. Acad. Sci. U.S.A. 101, 6462 (2004).

71. A. Yildiz, J.N. Forkey, S.A. McKinney, T. Ha, Y.E. Goldman, P.R. Selvin, Science 300, 2061 (2003)

72. R.E. Thompson, D.R. Larson, W.W. Webb, Biophys. J. 82, 2775 (2002).

73. M.L. Weber, K.A. Willets, J. Phys. Chem. Lett. 2, 1766 (2011).

74. S.M. Stranahan, K.A. Willets, Nano Lett. 10, 3777 (2010).

75. H. Cang, A. Labno, C. Lu, X. Yin, M. Liu, C. Gladden, Y. Liu, X. Zhang, Nature 469, 385 (2011).

76. D. Wu, Z. Liu, C. Sun, X. Zhang, Nano Lett. 8, 1159 (2008).

77. F.J. García de Abajo, Rev. Mod. Phys. 82, 209 (2010).

78. P.E. Batson, Phys. Rev. Lett. 49, 936 (1982).

79. J. Nelayah, M. Kociak, O. Stephan, F.J. García de Abajo, M. Tence, L. Henrard, D. Taverna, I. Pastoriza-Santos, L.M. Liz-Marzan, C. Colliex, Nat. Phys. 3, 348 (2007).

80. A.L. Koh, A.I. Fernández-Domínguez, D.W. McComb, S.A. Maier, J.K.W. Yang, Nano Lett. 11, 1323 (2011).

81. A. Yurtsever, R.M. van der Veen, A.H. Zewail, Science 335, 59 (2012).

82. J.A. Scholl, A.L. Koh, J.A. Dionne, Nature 483, 421 (2012).

83. J.T. van Wijngaarden, E. Verhagen, A. Polman, C.E. Ross, H.J. Lezec, H.A. Atwater, Appl. Phys. Lett. 88, 221111 (2006).

84. M.V. Bashevoy, F. Jonsson, A.V. Krasavin, N.I. Zheludev, Y. Chen, M.I. Stockman, Nano Lett. 6, 1113 (2006).

85. T. Coenen, E.J.R. Vesseur, A. Polman, Appl. Phys. Lett. 99, 143103 (2011). 86. C.E. Hofmann, E.J.R. Vesseur, L.A. Sweatlock, H.J. Lezec, F.J. Garcia de Abajo, A. Polman, H.A. Atwater, Nano Lett. 7, 3612 (2007).

87. M. Kuttge, E.J.R. Vesseur, A.F. Koenderink, H.J. Lezec, H.A. Atwater, F.J. García de Abajo, A. Polman, Phys. Rev. B 79, 2 (2009).

88. E.J.R. Vesseur, A. Polman, Nano Lett. 11, 5524 (2011).

89. M. Frimmer, T. Coenen, A. Koenderink, Phys. Rev. Lett. 108, 1 (2012).

90. T. Coenen, E.J.R. Vesseur, A. Polman, ACS Nano 6, 1742 (2012).

91. X.L. Zhu, Y. Ma, J.S. Zhang, J. Xu, X.F. Wu, Y. Zhang, X.B. Han, Q. Fu, Z.M. Liao, L. Chen, D.P. Yu, Phys. Rev. Lett. 105, 1 (2010).

92. A. Reyes-Coronado, R.G. Barrera, P.E. Batson, P.M. Echenique, A. Rivacoba, J. Aizpurua, Phys. Rev. B 82, 235429 (2010).

93. P.E. Batson, A. Reyes-Coronado, R.G. Barrera, A. Rivacoba, P.M. Echenique, J. Aizpurua, Nano Lett. 11, 3388 (2011).
94. E. Altewischer, M.P. van Exter, J.P. Woerdman, Nature 418, 304 (2002).

95. E. Moreno, F. García-Vidal, D. Erni, J. Cirac, L. Martín-Moreno, Phys. Rev. Lett. 92, 3 (2004).

96. S. Fasel, F. Robin, E. Moreno, D. Erni, N. Gisin, H. Zbinden, Phys. Rev. Lett. 94, 1 (2005).

97. R. Zia, M.L. Brongersma, Nat. Nanotechnol. 2, 426 (2007).

98. R. Kolesov, B. Grotz, G. Balasubramanian, R.J. Stoehr, A.A.L. Nicolet, P.R. Hemmer, F. Jelezko, J. Wrachtrup, Nat. Phys. 5, 470 (2009).

99. A.V. Akimov, A. Mukherjee, C.L. Yu, D.E. Chang, A.S. Zibrov, P.R. Hemmer, H. Park, M.D. Lukin, Nature 450, 402 (2007).

100. D.E. Chang, A.S. Sørensen, E.A. Demler, M.D. Lukin, Nat. Phys. 3, 807 (2007)

101. F.H.L. Koppens, D.E. Chang, F.J. García de Abajo, Nano Lett. 11, 3370 (2011).

102. R. Esteban, A.G. Borisov, P. Nordlander, J. Aizpurua, Nat. Commun. 3, 825 (2012).

103. C.L. Cortes, W. Newman, S. Molesky, Z. Jacob, J. Opt. 14, 063001 (2012). 104. H.N.S. Krishnamoorthy, Z. Jacob, E. Narimanov, I. Kretzschmar, V.M. Menon, Science 336, 205 (2012)

105. E. Yablonovitch, 10th IEEE International Conference on Nanotechnology 13 (2010), doi:10.1109/NAN0.2010.5697734.

106. S. Xiao, V.P. Drachev, A.V. Kildishev, X. Ni, U.K. Chettiar, H.-K. Yuan, V.M. Shalaev, Nature 466, 735 (2010).

107. J.B. Khurgin, G. Sun, Appl. Phys. Lett. 96, 181102 (2010)

108. P. Tassin, T. Koschny, M. Kafesaki, C.M.A. Soukoulis, Nat. Photonics 6. 259 (2012)

109. G. Naik, J. Kim, A. Boltasseva, Opt. Mater. Express 1, 1090 (2011).

110. G. Naik, J.L. Schroeder, X. Ni, A.V. Kildishev, T.D. Sands, A. Boltasseva, Opt. Mater. Express 2, 478 (2012).

111. K. Manthiram, A.P. Alivisatos, J. Am. Chem. Soc. 134, 3995 (2012).

112. J.M. Luther, P.K. Jain, T. Ewers, A.P. Alivisatos, Nat. Mater. 10, 361

(2011).

113. G. Garcia, R. Buonsanti, E.L. Runnerstrom, R.J. Mendelsberg, A. Llordes, A. Anders, T.J. Richardson, D.J. Milliron, Nano Lett. 11, 4415 (2011).

114. Z. Fei, A.S. Rodin, G.O. Andreev, W. Bao, A.S. McLeod, M. Wagner, L.M. Zhang, Z. Zhao, M. Thiemens, G. Dominguez, M.M. Fogler, A.H. Castro Neto, C.N. Lau, F. Keilmann, D.N. Basov Nature (2012), doi:10.1038/nature11253 115. E.N. Economou, Phys. Rev. 182, 539 (1969).

116. E. Kretschmann, H. Raether, Z. Naturforsch. A 23, 2135 (1968).

117. U. Kreibig, M. Vollmer, Optical Properties of Metal Clusters (Springer, NY, 1995).

118. A. Nevet, N. Berkovitch, A. Hayat, P. Ginzburg, S. Ginzach, O. Sorias, M. Orenstein, Nano Lett. 10 (5), 1848 (2010).

119. J. Chen, M. Badioli, P. Alonso-González, S. Thongrattanasiri, F. Huth, J. Osmond, M. Spasenović, A. Centeno, A. Pesquera, P. Godignon, A. Zurutuza Elorza, N. Camara, F.J. García de Abajo, R. Hillenbrand, F.H.L. Koppens, Nature (2012), doi:10.1038/nature11254.

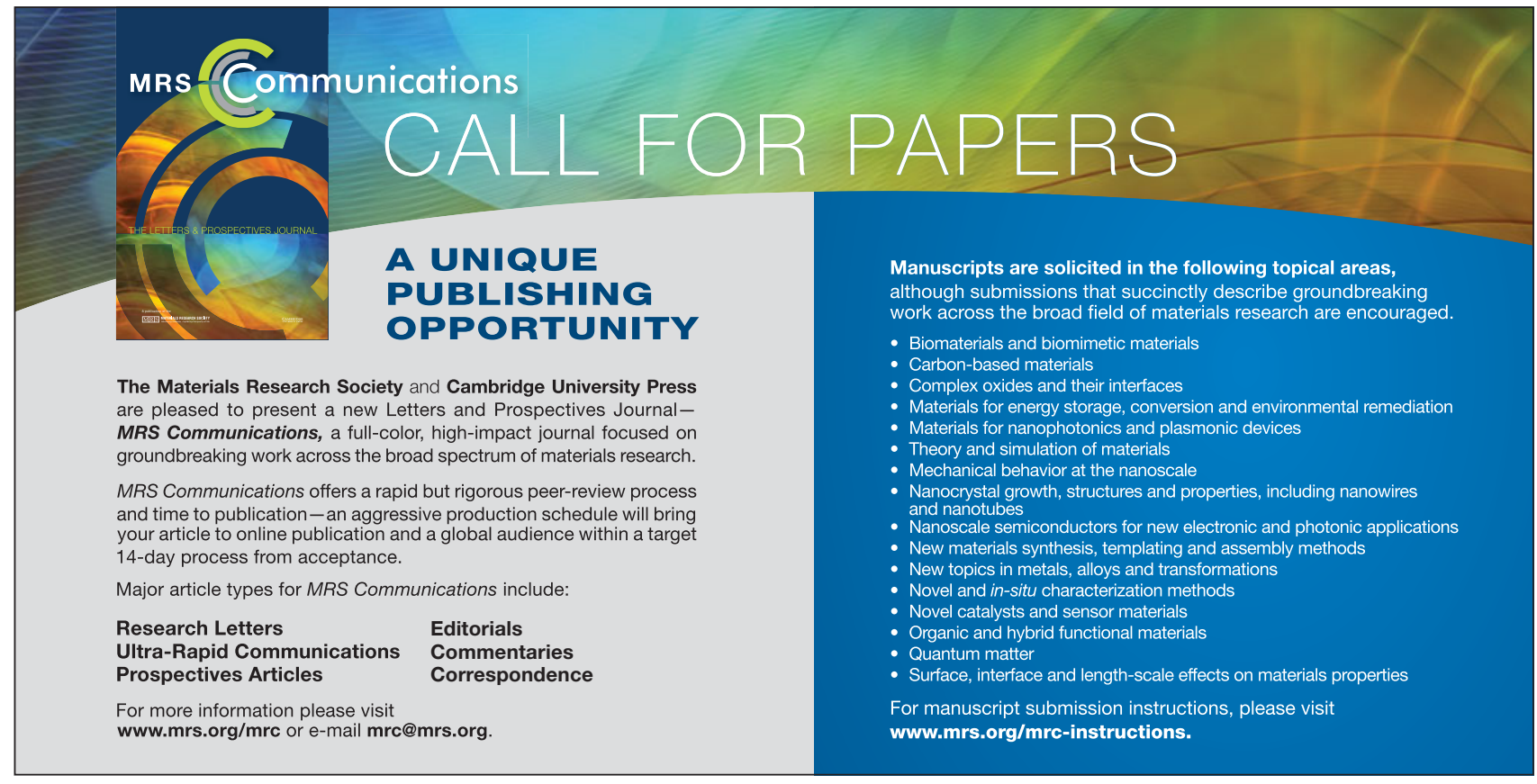

